Nota Científica

(Short communication)

\title{
SERPIENTES ASOCIADAS A UN ÁREA URBANA EN EL DEPARTAMENTO DE CÓRDOBA, COLOMBIA
}

\author{
SNAKES ASSOCIATED TO AN URBAN AREA IN THE CORDOBA DEPARTMENT, \\ COLOMBIA
}

\author{
JosÉ G. VILORIA-RIVAS* \& JULIO J. CHACÓN-PACHECO \\ Grupo de Investigación Biodiversidad Unicórdoba, Facultad de Ciencias Básicas, Departamento de Biología. Universidad de \\ Córdoba. Carrera 6 No. 76-103, Montería, Córdoba, Colombia. Código Postal: 230002.<jchacon_bio@hotmail.com> \\ *Autor de correspondencia: <viloriarivas@hotmail.com>
}

Recibido: 01/09/2017; aceptado: 26/01/2018; publicado en línea: 23/10/2018

Editor responsable: Gustavo Aguirre

Viloria-Rivas, J., Chacón-Pacheco, J. (2018) Serpientes asociadas a un área urbana en el departamento de Córdoba, Colombia. Acta Zoológica Mexicana (nueva serie), 34, 1-4. DOI: https://doi.org/10.21829/azm.2018.3412108

RESUMEN. Se determinó la riqueza de serpientes asociada a un área urbana, la Universidad de Córdoba ubicada en la ciudad de Montería, departamento de Córdoba, Colombia. Entre enero de 2011 y noviembre de 2014, se aplicaron capturas a través de trampas de caída, búsqueda libre restringida por tiempo y búsqueda libre. Se registraron diez especies, distribuidas en diez géneros y cuatro familias, la familia Didapsidae fue la mejor representada con el $60 \%$ de las especies reportadas, seguida de la familia Colubridae $20 \%$, Boidae y Leptotyphlopidae con el $10 \%$ cada una. Los ofidios en esta localidad se han visto afectados por el temor del personal administrativo y los trabajadores de la Universidad, debido a la falta de conocimiento sobre la ecología y el comportamiento de las serpientes.

Viloria-Rivas, J., Chacón-Pacheco, J. (2018) Snakes associated to an urban area in the Córdoba Department, Colombia. Acta Zoológica Mexicana (nueva serie), 34, 1-4. DOI: https://doi.org/10.21829/azm.2018.3412108

ABSTRACT. The objective of this study was to determine the richness of snake species associated to an urban area, the University of Cordoba in the city of Montería, Colombia. The study was conducted between January 2011 and October 2014. Ten species distributed in ten genera and four families were recorded, the family Dipsadidae was represented with 60\%, Boidae and Leptotyphlopidae with $10 \%$ each one. The progressive increase of construction activities and suitability of land for crops have contributed to the loss of habitat for many species of snakes. In addition, in this locality these reptiles have been affected by the fear of the administrative personnel and the workers of the University, due to the lack of knowledge on the ecology and the behavior of these organisms.

El departamento de Córdoba es privilegiado por presentar gran diversidad de especies y de ambientes climáticos (Carvajal-Cogollo et al., 2007; Ballesteros \& Linares, 2015). Los estudios sobre la diversidad de reptiles han sido enfatizados en ambientes naturales (Carvajal-Cogollo et al., 2007, Ruiz-Pinto, 2005; Carvajal-Cogollo \& Urbina-Cardona, 2008; Bernal \& Nonzoque, 2010; Cárdenas-Arévalo et al., 2010; 
Ruiz-Pinto, 2014), obviando la diversidad presente en ambientes con influencia antropogénica y más aún en grupos tan estigmatizados por los humanos como son los ofidios.

En relación a lo anterior, conocer la riqueza de serpientes en ambientes perturbados, particularmente en la Universidad de Córdoba, se convierte en una buena herramienta para la conservación de serpientes y de los hábitats explotados por las mismas. Así mismo, el aumento del conocimiento sobre la ecología de estas especies contribuye a la toma de mejores decisiones relacionadas con el manejo de ofidios. Este trabajo buscó conocer las especies de serpientes presentes en el campus de la Universidad de Córdoba (847'26.5"N, 7551'39.6"W), la cual se encuentra ubicada en la ciudad de Montería, departamento de Córdoba, Colombia. La Universidad presenta un área de 57 ha que limita con el Río Sinú, se encuentra ocupada en su mayoría por zonas agrícolas (Cu) (847'33.7"N, 7551'34.1"W), piscícolas (Ep) (847'30.3"N, 7551'50.3"W), inmuebles (edificaciones, aulas y cubículos de un solo piso), zonas verdes y vegetación riparia $(\mathrm{Vr})\left(8^{\circ} 47^{\prime} 25.8^{\prime \prime} \mathrm{N}, 75^{\circ} 51^{\prime} 55.1^{\prime \prime} \mathrm{W}\right)$ con altos grados de modificación dada la tala selectiva y establecimiento de árboles maderables como son Tectona grandis y Tabebuia rosea (Fig. 1).
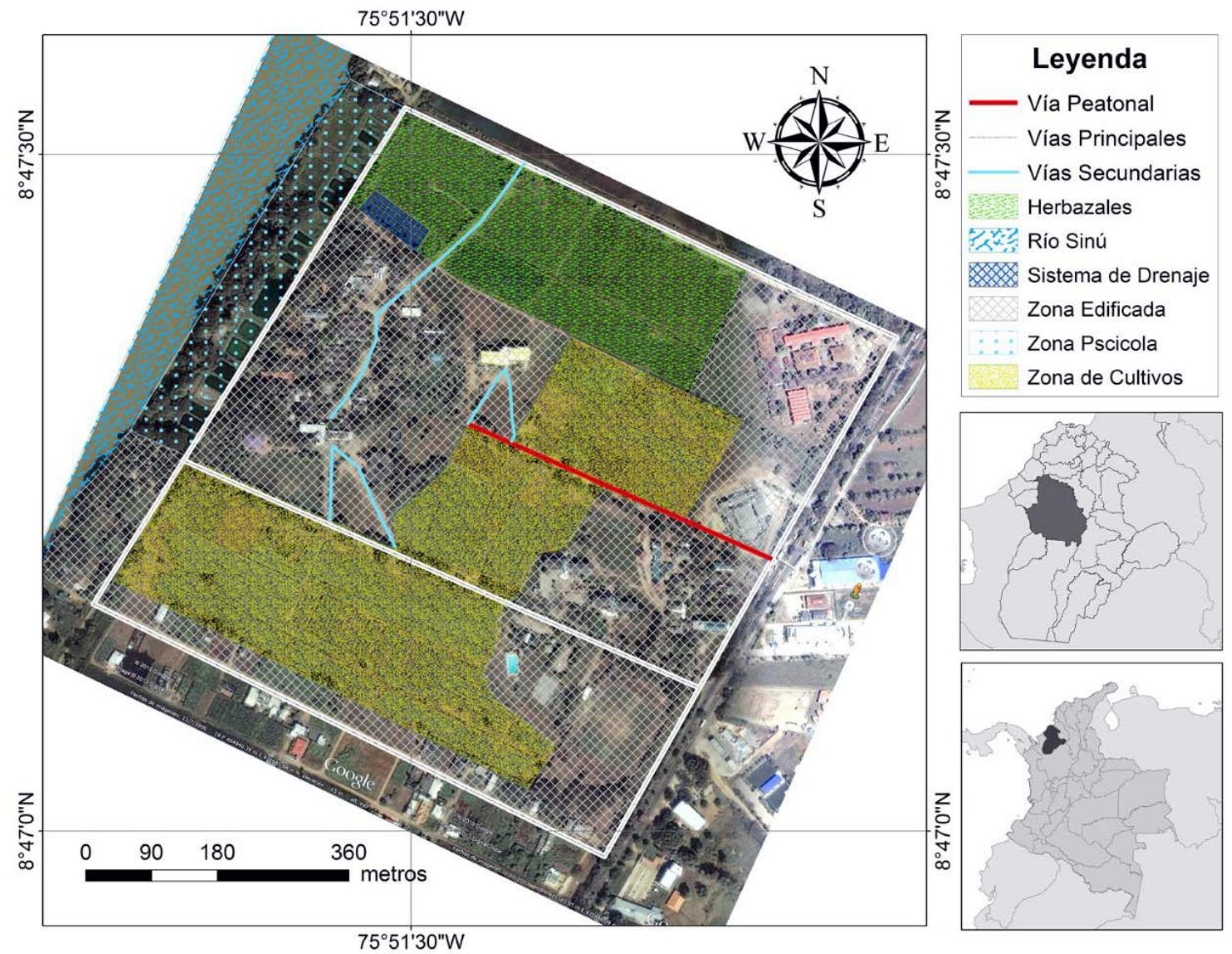

Figura 1. Localización y descripción del área de estudio, Universidad de Córdoba, Montería - Córdoba, Colombia.

En este trabajo se realizaron muestreos aleatorios con estudiantes de los programas de Biología y Medicina Veterinaria y Zootecnia, en el marco de capacitaciones en métodos de colecta de vertebrados silvestres, entre enero de 2011 y noviembre de 2014 empleando los siguientes métodos de muestreo: trampas de caída, búsqueda libre restringida por tiempo y búsqueda libre, tratando de abarcar la mayor 
cantidad de hábitats y microhábitats presentes en los sitios de muestreo (Angulo et al., 2006). Posterior a la captura, los individuos se identificaron con la ayuda de claves taxonómicas (Pérez-Santos, 1986; Castro-Herrera, 2008; Hedges, 2011).

Se encontraron diez especies, pertenecientes a nueve géneros y cuatro familias (Cuadro 1), lo que constituye el 3.70\% de las especies registradas para Colombia (Lynch, 2012) y el 19.23\% para el departamento de Córdoba (Ballesteros \& Linares, 2015). La riqueza de especies encontrada puede atribuirse a los atributos ecológicos de las especies como vagilidad y plasticidad en la utilización de microhábitats (Urbina-Cardona et al., 2008), expresado en el registro de estas especies principalmente para las zonas de cultivo (CU) y la estación piscícola (EP), zonas que se reconocen por la oferta alimenticia (roedores y anfibios). Para Córdoba, a pesar de reportarse con frecuencia especies del género Thamnodynastes, tanto en zonas inundadas como altamente intervenidas (Carvajal-Cogollo et al., 2007; Ballesteros \& Linares, 2015), para este estudio no se hicieron registros tal vez debido a la asociación de estas especies a arbustos y pajonales alrededor de grandes cuerpos de agua, como ciénagas o pantanos (Rengifo \& Lundberg, 1999).

Cuadro 1. Especies de serpientes asociadas a ambientes antropizados en la Universidad de Córdoba, Colombia. Cu: Cultivos; EP: Estación Piscícola; Ed: Edificaciones; VR: Vegetación riparia.

\begin{tabular}{lll}
\hline Familia & Especie & Lugar de captura \\
\hline Boidae & Epicrates cenchria & $\mathrm{Cu}, \mathrm{EP}$ \\
Colubridae & Tantilla melanocephala & $\mathrm{Ed}$ \\
& Enulius flavitorques & $\mathrm{EP}$ \\
Dipsadidae & Helicops danielii & $\mathrm{Cu}, \mathrm{EP}, \mathrm{Ed}$ \\
& Leptodeira septentrionalis & $\mathrm{Cu}, \mathrm{EP}, \mathrm{VR}$ \\
& Lygophis lineatus & $\mathrm{EP}, \mathrm{Ed}, \mathrm{Cu}$ \\
& Erythrolamprus melanotus & $\mathrm{EP}, \mathrm{Ed}, \mathrm{Cu}$ \\
& Pseudoboa neuwiedii & $\mathrm{Cu}$ \\
& Oxyrhopus cf. petolarius & $\mathrm{VR}$ \\
Leptotyphlopidae & Trilepida macrolepis & $\mathrm{EP}$ \\
\hline
\end{tabular}

Es importante resaltar que especies como Epicrates cenchria, Helicops danielii y Leptodeira septentrionalis, se han visto afectadas en el campus de la Universidad de Córdoba, dado que autoridades administrativas ordenan al personal de mantenimiento la caza de las serpientes, a pesar de que en el campus no se han registrado especies que representen un peligro significativo para los humanos. Helicops danielii y L. septentrionalis usualmente están sujetas a la creencia popular de poseer venenos altamente tóxicos, asociándolas al género Bothrops (mapanas), las cuales si suponen peligro para el hombre. Sin embargo, es de aclarar que la especie semivenenosa $L$. septentrionalis, por su naturaleza opistoglifa, segrega sustancias que podrían ser catalogadas como tóxicas, dadas sus propiedades hemotóxicas, neurotóxicas y proteolíticas (Lemoine et al., 2004), pero no se registran muertes ni alteraciones fisiopatológicas relevantes por su mordedura (Ruiz-Pinto, 2014).

Dada la relación hombre-serpiente en ambientes urbanos como es el caso del campus de la Universidad de Córdoba, expresada en la ocupación de espacios comunes y el temor de los humanos hacia estos animales (Casas-Andreu, 2000), se hace necesario implementar programas de educación y aspectos de seguridad sobre el manejo y atención de accidentes ofídicos. 
Agradecimientos. A los estudiantes de Biología y Medicina Veterinaria que participaron en el trabajo de campo. A Faidith Bracho-Altamiranda por su revisión crítica del documento.

\section{LITERATURA CITADA}

Casas-Andreu, G. (2000) Mitos, leyendas y realidades de los reptiles en México. Ciencia Ergo Sum, 7, 286-291.

Angulo, A., Rueda-Almonacid, J.V., Rodríguez-Mahecha, J.V., La Marca, E. (2006) Técnicas de inventario y monitoreo para los anfibios de la región tropical andina. Conservación Internacional. Bogotá, Colombia. 300 pp.

Ballesteros, J., Linares, J. (2015) Fauna de Córdoba, Colombia. Grupo Investigación Biodiversidad UniCórdoba, Facultad de Ciencias Básicas. Fondo Editorial, Universidad de Córdoba. Bogotá. $324 \mathrm{pp}$.

Bernal, V., Nonzoque, N. (2010) Diversidad del ensamble de serpientes (Squamata: Serpentes) en elementos del paisaje del sur del departamento de Córdoba-Colombia. Universidad Pedagógica y Tecnológica de Colombia. Tunja, Colombia.

Cárdenas-Arévalo, G., Castaño-Mora, O., Carvajal-Cogollo, J., Rangel-Ch, J. (2010) Comunidad de reptiles en humedales y áreas aledañas del departamento de Córdoba. Pp. 361-380. In: J. Rangel (Ed.). Colombia diversidad biótica IX: ciénagas de Córdoba: biodiversidad-ecología y manejo ambiental. Instituto de Ciencias Naturales-Universidad Nacional de Colombia, Bogotá, Colombia.

Carvajal-Cogollo, J. E., Castaño-Mora, O. V., Cárdenas-Arévalo, G., Urbina-Cardona, J. N. (2007) Reptiles de áreas asociadas a humedales de la planicie del departamento de Córdoba, Colombia. Caldasia, 29, 427-438.

Carvajal-Cogollo, J. E., Urbina-Cardona, J. N. (2008) Patrones de diversidad y composición de reptiles en fragmentos de bosque seco tropical en Córdoba, Colombia. Tropical Conservation Science, 1, 397-416.

Castro-Herrera, F. (2008) Taxonomía de Grupos Mayores de Anfibios y Reptiles Colombianos. Universidad del Valle. Cali, Colombia. 88 pp.

Hedges, S. B. (2011) The type species of the threadsnake genus Tricheilostoma Jan revisited (Squamata, Leptotyphlopidae). Zootaxa, 3027, 63-64.

Lemoine, K., Girón, M. E., Aguilar, I., Navarrete, L. F., Rodríguez-Acosta, A. (2004) Proteolytic, hemorrhagic, and neurotoxic activities caused by Leptodeira annulata ashmeadii (Serpentes: Colubridae) Duvernoy's gland secretion. Wilderness \& Environmental Medicine, 15, 82-89.

Lynch, J. D. (2012) El contexto de las serpientes de Colombia con un análisis de las amenazas en contra de su conservación. Revista de la Academia Colombiana de Ciencias Exactas, Físicas y Naturales, 36, 435-449.

Pérez-Santos, C. (1986) Las serpientes del Atlántico. Museo Nacional de Ciencias Naturales. Madrid, España. 83 pp.

Rengifo, J. M., Lundberg, M. (1999) Guía de campo Anfibios y Reptiles de Urrá. Ed 1a, proyecto Skanska, p. 58-62.

Ruiz-Pinto, O. (2014) Ofidios del corregimiento de San Rafael de Pirú, Valencia, Córdoba - Colombia. Revista Colombiana de Ciencia Animal, 6, 3-13.

Ruiz-Pinto, O. (2005). Caracterización preliminar de la herpetofauna reptiliana de las ciénagas de Betancí, Martinica y Nariño, pertenecientes a la cuenca del Río Sinú, Colombia. Trabajo de grado. Universidad de Córdoba. Montería, Colombia.

Urbina-Cardona, J. N., Londoño-Murcia, M. C., García-Ávila, D. G. (2008) Spatio-temporal dynamics of snake diversity in four habitats with different degrees of anthropogenic disturbance in the Gorgona Island National Natural Park in the Colombian Pacific. Caldasia, 30, 479-493. 